\title{
An approximate method of short-term tsunami forecast and the hindcasting of some recent events
}

\author{
Yu. P. Korolev \\ Institute of Marine Geology and Geophysics, Far East Branch, Russian Academy of Sciences, 1b Nauki Str., \\ Yuzhno-Sakhalinsk, 693022, Russia \\ Received: 3 October 2010 - Revised: 5 October 2011 - Accepted: 6 October 2011 - Published: 28 November 2011
}

\begin{abstract}
The paper presents a method for a short-term tsunami forecast based on sea level data from remote sites. This method is based on Green's function for the wave equation possessing the fundamental property of symmetry. This property is well known in acoustics and seismology as the reciprocity principle. Some applications of this principle on tsunami research are considered in the current study. Simple relationships and estimated transfer functions enabled us to simulate tsunami waveforms for any selected oceanic point based only on the source location and sea level data from a remote reference site. The important advantage of this method is that it is irrespective of the actual source mechanism (seismic, submarine landslide or other phenomena). The method was successfully applied to hindcast several recent tsunamis observed in the Northwest Pacific. The locations of the earthquake epicenters and the tsunami records from one of the NOAA DART sites were used as inputs for the modelling, while tsunami observations at other DART sites were used to verify the model. Tsunami waveforms for the 2006, 2007 and 2009 earthquake events near Simushir Island were simulated and found to be in good agreement with the observations. The correlation coefficients between the predicted and observed tsunami waveforms were from 0.50 to 0.85 . Thus, the proposed method can be effectively used to simulate tsunami waveforms for the entire ocean and also for both regional and local tsunami warning services, assuming that they have access to the real-time sea level data from DART stations.
\end{abstract}

\section{Introduction}

A short-term tsunami forecast and effective tsunami warning is a key problem of the tsunami service. At present, the conventional method for short-term tsunami forecasting is based

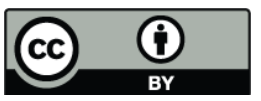

Correspondence to: Yu. P. Korolev (yu_p_k@mail.ru) on seismic information only (earthquake magnitude, time of the main shock and epicenter location). This method provides the fastest indicator of the tsunamigenic potential of the earthquake; it also enables us to quickly estimate the expected tsunami arrival time. Tsunami alert criteria are dependent on the exact source region and on historical tsunami impacts (Igarashi et al., 2011). An earthquake magnitude which exceeds the established threshold value, which is different for different tsunamigenic zones, typically results in the issuing of a Tsunami Warning (Gusiakov, 2011). This approach, based on a "magnitude-geographical principle" is straightforward and rather effective: at least it ensures few tsunami omissions. However, this method cannot provide sufficiently accurate estimates of tsunami wave heights, especially for specific coastal areas. Firstly, this is because the dependence of the tsunami intensity on the earthquake magnitude is far from being deterministic (Gusiakov, 2011): strong earthquakes can produce weak tsunamis (or no tsunamis at all) and, vice versa, sometimes destructive tsunamis are induced by relatively weak earthquakes. Secondly, because actual tsunami runups are strongly variable along the coast (cf. MacInnes et al., 2009), the tsunami alarm can be justified for one coastal site but false for another one. Moreover, the magnitude-geographical warning method does not allow evaluation of the duration of the tsunami alarm or prediction of the arrival time of the largest tsunami wave (not necessary the first, which is quite typical for tsunami events, cf. Rabinovich et al., 2008). Consequently, up to $75 \%$ of tsunami warnings turn out to be false (Gusiakov, 2011). False tsunami warnings cause anxiety among emergency managers, government officials and the business community. These false alarms result in actual financial losses, sometimes significant ${ }^{1}$, including losses due to production downtime, and

\footnotetext{
1 The cost of the false alarm associated with the 1994 Shikotan tsunami for only the Hawaiian Islands was two human lives (one during the evacuation response and the other due to a heart attack) and about US\$ 30 million dollars in economic loss (Rabinovich et al., 2006; also L. Kong, personal communication, 2008).
}

Published by Copernicus Publications on behalf of the European Geosciences Union. 
expenses for the emergency evacuation procedures and navigation of vessels to the open sea (NOAA Magazine, 2011).

According to the modern concept, a tsunami alarm should be declared timely and selectively only for exposed coastal areas where the incoming tsunami will be of a real threat. Each tsunami alarm should be accompanied by trustworthy information on the expected tsunami arrival time, wave heights along the coast, their frequencies, and the duration for dangerous oscillations to remain in effect. However, based merely on the seismological data such information cannot be obtained. Meanwhile, emergency managers and local officials are in urgent need of effective operational tools that can provide reliable tsunami forecasts as guidance for rapid, critical decisions in which lives and property are at stake (Titov et al., 2005).

From this point of view, direct tsunami monitoring in the open ocean by DART ${ }^{2}$ stations can significantly improve the situation (Titov et al., 2005; Titov, 2009). These deep-ocean real-time tsunameters have been developed by the Pacific Marine Environmental Laboratory (PMEL/NOAA) for the early detection, measurement, and real-time reporting of farfield tsunamis. These stations have been deployed at strategic locations throughout the Pacific Ocean near the main seismically active regions with a history of tsunami generation (Mofjeld, 2009). DART tsunami monitoring systems were expected to become a backbone of the modern Tsunami Warning System (TWS) in the Pacific Ocean.

The principal methodology of the tsunami forecast based on open-ocean sea level measurements had been developed during 1985-2005 (cf. Satake, 1987; Chubarov and Shokin, 1995; Korolev and Poplavsky, 1995; Poplavsky et al., 1997; Voronina and Tcheverda, 1998; Wei et al., 2003; Titov et al., 2005; Yamazaki et al., 2006). However, only recent advances in tsunami measurement and numerical modeling technology made it possible to create effective tsunami forecasting systems. The tsunami forecasting technology elaborated at PMEL is based on the integration of realtime DART measurements and state-of-the-art numerical modeling (Titov et al., 2005). This technology was applied in an operative regime for several 2005-2011 major events and demonstrated to be highly efficient (cf. Tang et al., 2008; Wei et al., 2008; Titov, 2009). The maximum wave heights and other parameters of arriving tsunami for several US sites were predicted and were later found to be in good agreement with the actual in situ observed waves (Titov, 2009).

It should be noted, however, that the PMEL technology is based on a pre-computed Propagation Database to compute a quick preliminary forecast of the ocean-wide propagation of the tsunami as a linear combination of unit sources selected to represent the initial earthquake parameters. Once the actual tsunami wave reaches a DART station, inversion is

\footnotetext{
${ }^{2}$ DART $=$ Deep-ocean Assessment and Reporting of Tsunami is a deep-sea tsunami monitoring system by the US National Oceanic and Atmospheric Administration (NOAA).
}

performed to adjust the slip distribution of the selected unit sources. The Propagation Database is a key component of the operational tsunami forecasting system (known as SIFT). At present, it includes 1691 pre-computed model runs for simulated earthquake sources in the Pacific, Atlantic and Indian oceans (Titov, 2009; see also http://nctr.pmel.noaa.gov/ propagation-database.html).

In the present study we suggest a simple method for short-term tsunami forecasting that does not require precomputed tsunami waveforms. The method is based on a reciprocity principle (cf. Rayleigh, 1945; Loomis, 1979) and uses only the seismological information about co-ordinates of the earthquake epicenter and in situ tsunami data from one of the far-field open-ocean DART stations. This method can work in a real-time mode and, therefore, be effectively used for local Tsunami Warning.

\section{Reciprocity principle}

The reciprocity principle for harmonic waves in acoustics has been known for more than $140 \mathrm{yr}$, since the pioneer studies of Hermann von Helmholtz and Lord Rayleigh (cf. Rayleigh, 1945). It is a corollary of the properties of symmetry of Green's function for a linear wave equation. The validity of the reciprocity principle for an inhomogeneous medium with reflecting, absorbing and mixed (impedance) boundaries was proved later. It is valid for any areas, including shadow zones, and including the case of non-stationary waves (Chertock, 1970). The reciprocity principle is widely applied in hydroacoustics for hydrophone calibration (Uric, 1975). The first use of this principle for long water waves was probably suggested by Loomis $(1974,1979)$. The reciprocity principle was found to be effective to optimize tsunami precomputations. However, the effects of the ocean depth inhomogeneity had not been analysed previously. In view of large horizontal scales of tsunami sources, these effects can be significant.

Let's assume that a wave's process is described by the linear shallow-water equations: Source $T$ is located within area $S_{T}$ with the earthquake epicenter at point $T$ with coordinates $\left(x_{T}, y_{T}\right)$, while Source $A$ is within area $S_{A}$ with the epicenter at $A$ (coordinates $x_{A}, y_{A}$ ). For simplicity, the initial sea surface elevation is used as the wave source.

It is not difficult to demonstrate, following the algorithm described by Brekhovskikh (1960) and Landau and Lifshits (1987), that for water waves from two distant sources $A$ and $T$ in areas $S_{A}$ and $S_{T}$, the following exact relationship is true: 


$$
\begin{aligned}
& \int_{S_{A}} \zeta_{A 0}(x, y) \times \zeta_{T}(s, x, y) \times d x d y \\
& =\int_{S_{T}} \zeta_{T 0}(x, y) \times \zeta_{A}(s, x, y) \times d x d y,
\end{aligned}
$$

where $\zeta_{A 0}(x, y), \zeta_{T 0}(s, x, y)$ are the initial elevations of the free surface within areas $S_{A}$ and $S_{T}, \zeta_{T}(s ; x, y)$ is the Laplace transform of the waveform from Source $T$ in area $S_{A}$, while $\zeta_{A}(s ; x, y)$ is the Laplace transform of the waveform from Source $A$ in area $S_{T}$. The relationship (1) and the following reciprocity relationships are true in inhomogeneous media if the processes are linear and boundaries are reflecting and/or absorbing.

The classical statement of the reciprocity principle requires the following conditions to be satisfied:

a. The distance between the sources has to be longer than the wavelengths ( $x, y$ scales of the source areas), i.e. the variation $\Delta \zeta_{A}$ should be significantly smaller than function $\zeta_{A}(s ; x, y)$ within the area $S_{T}: \Delta \zeta_{A} \ll \zeta_{A}(s ; x, y)$. This should also be true for the function $\zeta_{T}(s ; x, y)$. An alternative asymptotic condition may be formulated in the time domain: typical wave periods should be significantly smaller than the time of wave propagation between the sources.

b. The horizontal $(x, y)$ scales of both source areas have to be shorter than the wave lengths of the generated tsunami waves.

Therefore, functions $\zeta_{A}(s ; x, y)$ and $\zeta_{T}(s ; x, y)$ in Eq. (1) can be removed from the integrals and replaced by mean values at points $T$ and $A$. Thus, an approximate expression that follows from Eq. (1) is:

$$
\begin{aligned}
& \zeta_{T}\left(s, x_{A}, y_{A}\right) \times \int_{S_{A}} \zeta_{A 0}(x, y) \times d x d y \approx \zeta_{A}\left(s, x_{T}, y_{T}\right) \\
& \times \int_{S_{T}} \zeta_{T 0}(x, y) \times d x d y .
\end{aligned}
$$

Designating

$$
\int_{S_{A}} \zeta_{A 0}(x, y) \times d x d y=Q_{A}
$$

and

$\int_{S_{T}} \zeta_{T 0}(x, y) \times d x d y=Q_{T}$, we can rewrite the last relationship as:

$\zeta_{T}\left(s ; x_{A}, y_{A}\right) \times Q_{A} \approx \zeta_{A}\left(s ; x_{T}, y_{T}\right) \times Q_{T}$,

where $Q_{A}$ and $Q_{T}$ are the volumes of the initial disturbances.

Due to the medium inhomogeneity (variable speed of wave propagation) and large horizontal source scales in comparison with the water depth, the initial free surface elevations should satisfy to the following conditions:

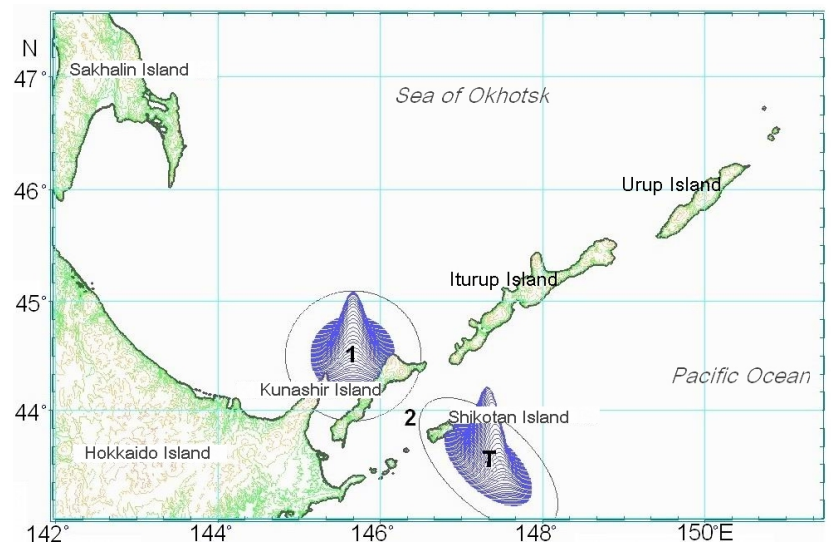

Fig. 1. A map showing the region of the South Kuril Islands and a scheme of numerical experiments. Symbols 1, 2 and $T$ indicate the epicenters of the sources. Initial sea surface elevations at Source 1 and Source $T$ are pictured.

- the forms of the initial disturbances should be similar;

- the disturbance radii $R_{A}$ and $R_{T}$ are related as

$\frac{R_{A}}{\sqrt{g D_{A}}}=\frac{R_{T}}{\sqrt{g D_{T}}}$,

where $D_{A}$ and $D_{T}$ are the water depths at points $A$ and $T$ and $g$ is the gravitational acceleration.

The approximate relationship (2) and the condition of similarity Eq. (3) are known as dynamic reciprocity principle (relationships) for non-stationary long water waves (Poplavsky et al., 1997).

Additionally, if the disturbance volumes are identical $\left(a_{A} \times R_{A}^{2}=a_{T} \times R_{T}^{2}\right.$, i.e. $\left.Q_{A}=Q_{T}\right)$, then after the inverse Laplace transform the waveforms also will match each other:

$\zeta_{T}\left(t, x_{A}, y_{A}\right)=\zeta_{A}\left(t, x_{T}, y_{T}\right)$

It is of interest to identify whether the relationship (4) is fair if conditions (a) and (b) are not satisfied. This is the subject of a study represented below.

To verify this approach we made several numerical experiments. The numerical simulations based on actual bathymetry were conducted for the region of the Southern Kuril Islands. Source 1 was located in the Sea of Okhotsk northwest of Kunashir Island; Source 2 in YuzhnoKurilsk Strait, between Kunashir and Shikotan islands; and the Source $T$ was in the Pacific Ocean southeast of Shikotan Island. The computational domain for the numerical experiments indicating the location of the sources is shown in Fig. 1. The bathymetry grid for these experiments was chosen to have a spatial step $\Delta x=\Delta y=1000 \mathrm{~m}$.

The parameters of the initial free surface elevations were chosen taking into account the above-mentioned conditions. They were the following: 


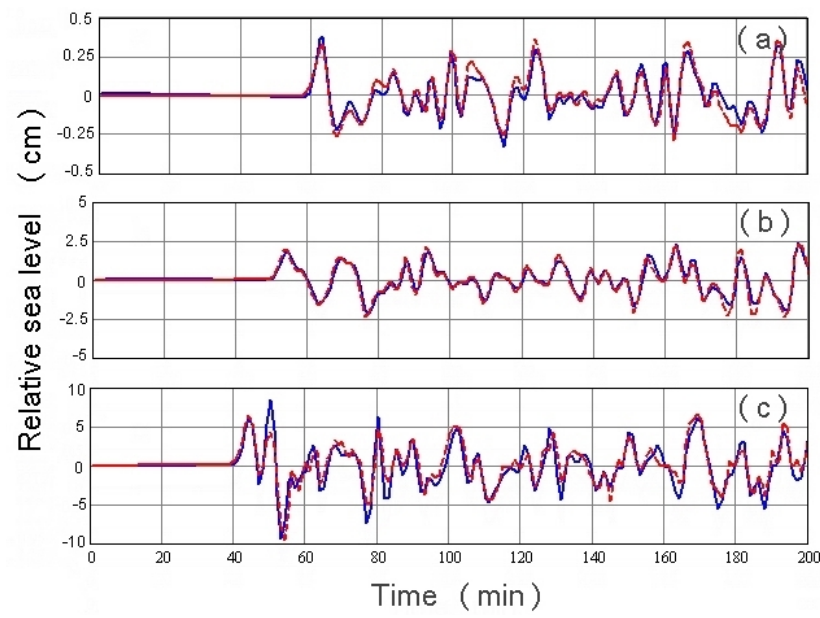

Fig. 2. Illustration of the reciprocity relationships. (a) Waveforms at Site $T$ due to Source 1 (blue solid line) and at Site 1 due to Source $T$ (red dashed line); correlation coefficient $\rho_{12}=0.96$. (b) Waveforms at Site 2 due to Source 1 (blue solid line) and at Site 1 due to Source 2 (red dashed line); correlation coefficient $\rho_{12}=0.94$. (c) Waveforms at Site $T$ due to Source 2 (blue solid) and at Site 2 due to Source $T$ (red dashed line) correlation coefficient $\rho_{12}=0.91$.

Source 1: $\max$ ampl. $a_{1}=0.4 \mathrm{~m}$, radius $R_{1}=25 \mathrm{~km}$, water depth in epicenter $D_{1}=2418 \mathrm{~m}$;

Source 2: max ampl. $a_{2}=15.6 \mathrm{~m}$, radius $R_{2}=4 \mathrm{~km}$, water depth in epicenter $D_{2}=63.6 \mathrm{~m}$;

Source $T$ : Max ampl. $a_{T}=0.49 \mathrm{~m}$, radius $R_{T}=$ $22.5 \mathrm{~km}$, water depth in epicenter $D_{T}=2030 \mathrm{~m}$.

The results of these simulations are shown in Fig. 2. The correlation coefficient, $\rho$, was chosen as a criterion for comparison of the reciprocal waveforms.

The coincidence of waveforms according to Eq. (4) is quite good despite wave sheltering by a chain of islands. The typical wave periods are about $10-18 \mathrm{~min}$; the time of wave propagation is $57 \mathrm{~min}$ between Sources 1 and $T, 50 \mathrm{~min}$ between Sources 1 and 2, and $40 \mathrm{~min}$ between Sources 2 and $T$. These propagation times are only three times longer than the wave periods. It is possible to make certain that wave lengths in the reciprocity source areas exceed their sizes. For example, the wavelength $\left(\lambda_{2}\right)$ in Source 2 for period of $T_{2}=18 \mathrm{~min}$ and water depth $D_{2}=63.6 \mathrm{M}$ is $\lambda_{2}=$ $T \times \sqrt{g D_{2}} \approx 27 \mathrm{~km}$, while the diameter of Source 2 is $8 \mathrm{~km}$.

Numerical experiments show that relationship (2) with condition (3) is true even if the distance between the sources is of the same order as the sizes of the source areas and if the wave lengths are comparable to or longer than these sizes. As was found from our experiments, changing the radius of one of the sources by $25 \%$ does not significantly influence the output.

Additional experiments were done when Sources 1 and 2 were the same, but Source $T$ was not a circle but an ellipse.
The ellipse minor $\left(b_{2}\right)$ and major $\left(b_{1}\right)$ axes were chosen as (1) $b_{1}=63.6 \mathrm{~km}$ and $b_{2}=31.8 \mathrm{~km}\left(b_{2} / b_{1}=1 / 2\right)$; and (2) $b_{1}=77.9 \mathrm{~km}$ and $b_{2}=26.0 \mathrm{~km}\left(b_{2} / b_{1}=1 / 3\right)$; maximum amplitude $a_{T}=0.49 \mathrm{~m}$ and water depth in epicenter $D_{T}=2030$ $\mathrm{m}$ (for both cases). We also changed the orientation of the major axis relative to true north. The condition of similarity of the source forms was not true, but it was accepted that $\frac{2 R_{A}}{\sqrt{g D_{A}}}=\frac{\sqrt{b_{1} \times b_{2}}}{\sqrt{g D_{T}}}$ and $4 a_{1} R_{1}^{2}=a_{T} b_{1} b_{2}$. The respective numerical results are shown in Fig. 3.

The correlation coefficients in all cases are in the range of 0.67 to 0.90 . The best result is in the case when the major axis was directed toward the north-west. This agreement is good enough and sufficient for any practical applications. The relationship (4) was found to be fair not only for circular sources but also for elliptic sources, at least for the moderate ratios of axes lengths.

\section{A method of short-term tsunami forecasting}

The classical statement of the reciprocity principle requires conditions (a) and (b) (see the previous section); however, our numerical experiments demonstrate that it can still be applied without these strict contingencies. The results presented below could become the basis for the short-term tsunami forecasting.

\subsection{The reciprocity principle and main computing relationships}

Korolev $(2004,2005)$ demonstrated that the derived reciprocity relationships could be efficiently applied for the problem of short-term (operative) tsunami forecasts based on sea level data from a remote open-ocean site. It is assumed that the reciprocity relationship (2) is true when one of the sources is natural, while the other source is artificial (numerical).

Let's assume Tsunami Source $T$, the initial sea surface elevation, is located within the area $S_{T}$. The total volume of the disturbed uplift is $Q_{T}$. Let us also assume that $M$ indicates the site of a sea level gauge (i.e. DART site) and $A$ the site of interest (a point for tsunami forecasting). The problem is to compute the tsunami waveform at site $A$ ("target site") using the sea level data at site $M$ ("reference site"). Mathematically, this problem does not have a unique solution. However, it can be considered as a "consumer's problem". Specific methods should be developed for solving this problem.

In this section, "waveform" means the Laplace (Fourier) transform of the waveform. Let the reciprocal sources be located within areas with epicentres at $A$ and $M$. Their disturbed volumes are $Q_{A}$ and $Q_{M}$, while $\eta_{A}\left(s ; x_{T}, y_{T}\right)$ and $\eta_{M}\left(s ; x_{T}, y_{T}\right)$ are the waveforms at site $T$ from reciprocal sources $S_{A}$ and $S_{M}$. For the natural tsunami waveform and 

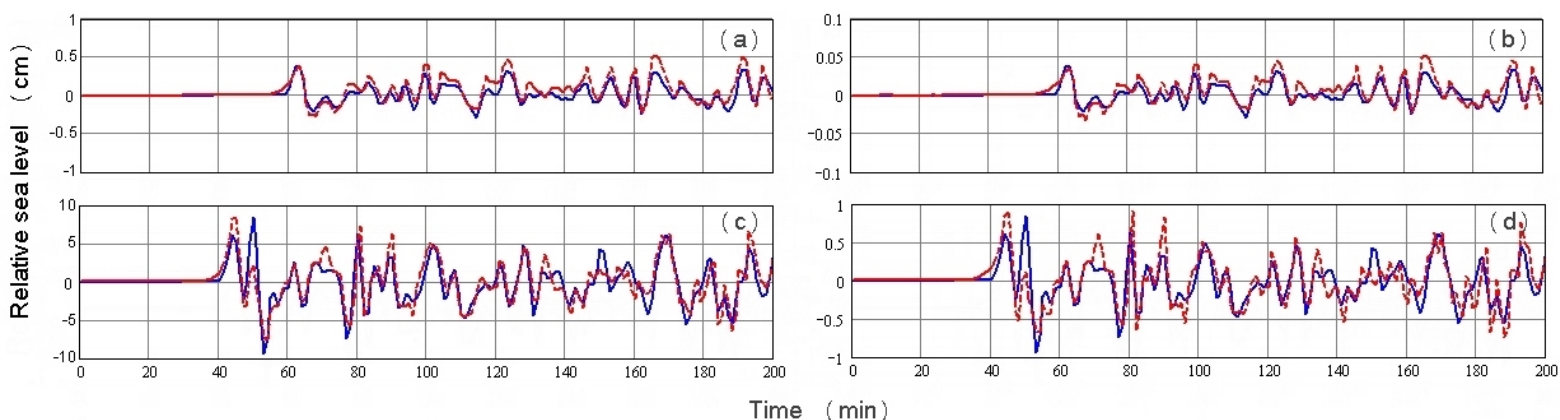

Fig. 3. Illustration of the reciprocity relationships. Elliptic Source $T$ is elongated to the north-west (see Fig. 1). The ratio of major/minor axes is equal to 2.0 (left column) and to 3.0 (right column). Upper row: waveforms at Site $T$ due to Source 1 (blue solid line) and at Site 1 due to Source $T$ (red dashed line); correlation coefficients are $\rho_{12}=0.90$ (a) and $\rho_{12}=0.87$ (b). Lower row: waveforms at Site $T$ due to Source 2 (blue solid line), and at Site 2 due to Source $T$ (red dashed line); correlation coefficients are $\rho_{12}=0.86$ (c) and $\rho_{12}=0.78$ (d).

for waveforms from the reciprocal sources in $S_{A}$ and $S_{M}$, relationships (2) are:

$$
\begin{aligned}
& \zeta_{T}(s, A) \times Q_{A}=\eta_{A}(s, T) \times Q_{T} ; \\
& \zeta_{T}(s, M) \times Q_{M}=\eta_{M}(s, T) \times Q_{T} .
\end{aligned}
$$

In these expressions we designated: $\zeta_{T}(s, A) \equiv \zeta_{T}\left(s, x_{A}, y_{A}\right)$, $\zeta_{T}(s, M) \equiv \zeta_{T}\left(s, x_{M}, y_{M}\right)$ and so on.

In addition, let's assume that the auxiliary sources are in the areas with the same epicenters at $A, M$ and $T$ (waveforms and disturbed volumes are designated by symbols with primes). For waveforms from these sources the reciprocity relationships are also true:

$$
\eta_{T}^{\prime}(s, A) \times Q_{A}^{\prime}=\eta_{A}^{\prime}(s, T) \times Q_{T}^{\prime}
$$$$
\eta_{T}^{\prime}(s, M) \times Q_{M}^{\prime}=\eta_{M}^{\prime}(s, T) \times Q_{T}^{\prime}
$$

As the auxiliary sources may be selected rather arbitrarily; in particular, $Q_{A}, Q_{M}$ and $Q_{A}^{\prime}, Q_{M}^{\prime}$ may be selected in such a way that $\eta_{A}^{\prime}$ is equal to $\eta_{A}$, and similarly for $\eta_{M}^{\prime}$ and $\eta_{M}$. It is true if $Q_{A}=Q_{A}^{\prime}$, and the same for Source $M$.

Eliminating unknown $Q_{T}$ from the above-mentioned two systems yields the following relationship:

$$
\frac{\zeta_{T}(s, A)}{\zeta_{T}(s, M)}=\frac{\eta_{T}(s, A)}{\eta_{T}(s, M)} .
$$

Let us assume that functions on the left side of the equation relate to one tsunami source, and functions on the right side relate to another source, and both sources have the same epicenter. The relationship (5) supports the well-known fact that spectra of any two tsunamis from different sources are similar when recorded in the same site, whereas spectra of any two tsunamis from the same source are dissimilar at different sites (cf. Takahasi and Aida, 1961; Miller, 1972).

From Eq. (5) we can get the following main relationship:

$\zeta_{T}(s, A)=\zeta_{T}(s, M) \frac{\eta_{T}(s, A)}{\eta_{T}(s, M)}$.
Here $\zeta_{T}(s, A)$ is the target function (tsunami waveform at $A) ; \zeta_{T}(s, M)$ is the reference function (tsunami waveform at $M$, i.e. open-ocean DART data); and $\eta_{T}(s, M)$ and $\eta_{T}(s, A)$ are the numerical waveforms at $M$ and $A$ from the auxiliary source located at $S_{T}$. All functions are Laplace transforms. The ratio on the right side of Eq. (6) plays the role of a transfer function.

It should be noted that the generation mechanism of the actual tsunami is not important here. The method can be applied for short-term forecasting irrespective of the tsunami source, in particular for tsunamis produced by earthquakes, volcanic eruptions, submarine landslides or other phenomena. However, for the practical realization of the proposed method it is important to assume that the transfer function for the corresponding event can be constructed as the ratio of the Laplace transforms of the waveforms at appropriate sites initiated by an initial free surface elevation. This elevation was supposed to be circular with a diameter of $50-100 \mathrm{~km}$ depending on the characteristic transversal size of the earthquake source. There is no requirement to know the detailed seismological information about the source; it is enough to know the coordinates of the earthquake (or another source) epicenter. This will enable us, with an accuracy sufficient for practical use, to create a short-term tsunami forecast for specific coastal sites.

The target tsunami waveform at site $A$, i.e. the left side function in expression (6), can be estimated after applying the inverse Laplace (Fourier) transform to Eq. (6) (Korolev, 2004). In fact, the proposed method is a generalization of the methods suggested earlier by Miller (1972), Chubarov and Shokin (1995) and Chung et al. (1995).

\subsection{Computing the short-term tsunami forecast}

The algorithm for computing the tsunami waveform based on sea level data from remote sites includes the following steps:

1. Deriving the coordinates of the earthquake (or other source) epicenter. 
2. Computing the tsunami waveforms for the reference site $M$ and the target (forecasting) site $A$, i.e. functions $\eta_{T}(s ; M)$ and $\eta_{T}(s ; A)$. The auxiliary source is a circle with diameter of $50-100 \mathrm{~km}$ located in the center of the actual tsunami source. The computation (evaluation of the transfer function) should be completed before the tsunami reaches the reference DART site (normally the site nearest to the source).

3. Acquiring the tsunami data from the appropriate reference DART station; eliminating tidal components from the corresponding record.

4. After recording and identifying tsunami waves at the reference DART station (Site $M$ ), the tsunami forecast can be made for the target site $A$ based on relationship (6). Similarly, in real-time mode, tsunami forecasts can be provided for other target sites (e.g. for particular sites along the coast). In fact, the preliminary forecast can be given after recording the first tsunami semi-wave. Hereafter, as new and more complete information is received, this forecast can be corrected and improved.

5. The forecast results can be used by TWCs for making their decision about declaring or cancelling the Tsunami Warning.

\section{Short-term tsunami forecast}

Some preliminary research results published in 2005, 2011 (Korolev, 2005; Korolev and Zaytsev, 2005; Korolev, 2011) demonstrated that the proposed method can be efficiently used for short-term tsunami forecasting. In particular, it was shown that the proposed method can work in a realtime mode and that the errors in the epicenter location only slightly affect the quality of the forecast.

\subsection{Preliminary information}

In the present study we apply this method to hindcast tsunamis waveforms for three tsunamigenic earthquakes that occurred in 2006, 2007 and 2009 in the region of the Central Kuril Islands, eastward from Simushir Island. Associated tsunamis were recorded by a number of tide gauges on the coasts of Russia and Japan (cf. Fujii and Satake, 2008; Rabinovich et al., 2008) and by several DART stations located along the Aleutian Islands and the US West Coast (Laverov et al., 2009; NOAA NDBC, 2011).

Tsunami waveforms were simulated for the locations of the DART stations. The actual in situ tsunami records data from the DART stations closest to the earthquake epicenters were used for the hindcast. The data from other DART stations, located further from the epicenter, were used to compare with the predictions and to verify the method. In the present study we used only deep-ocean stations, not affected by coastal resonant effects and surf beats. All computations were made based on the shallow-water numerical model described by Poplavsky et al. (1997). We used the Mercator projection with a spatial grid step of $3.8 \mathrm{~km}$ at latitude $40^{\circ} \mathrm{N}$. Bathymetric data were interpolated from the ETOPO2 global dataset (Smith and Sandwell, 1994). The correlation coefficient, $\rho$, was chosen as the criterion for comparison of the observed and predicted waveforms.

The scheme of the research experiments, indicating the earthquake epicenter and positions of the DART stations, are shown in Fig. 4. The hindcasting results are represented below.

\subsection{The November 2006 Simushir tsunami}

The tsunami was generated by a major earthquake $\left(M_{\mathrm{w}}=\right.$ 8.3) on 15 November 2006 with epicenter at $46.592^{\circ} \mathrm{N}$, $153.226^{\circ} \mathrm{E}$ near the Central Kuril Islands (NGDC Tsunami Database, 2011). Significant wave heights were observed along the coasts of the Kuril Islands (cf. Rabinovich et al., 2008; MacInnes et al., 2009). No seismological information about the earthquake, except the epicenter coordinates, was used in the following computations.

The auxiliary source with an initial circular sea surface uplift of $75 \mathrm{~km}$ in diameter and with a maximum height of $10 \mathrm{~m}$ was chosen as the input for the model. The selected source center coincided with the earthquake epicenter. The ocean depth in the epicenter, based on the bathymetry grid readings, was $2803 \mathrm{~m}$.

Figure 5 shows hindcast results and the waveforms of the observed tsunami waves. The computed waveforms match well the observed tsunami waveforms at DART stations 46402, 46403 and 46410 along the Aleutian Islands. Similar good agreement was observed also for DART stations 46419, 46411 and 46412 along the US West Coast. In all cases, the best agreement was for a few leading waves; the correlation coefficients for these waves were within the range $\rho=0.70-0.85$.

\subsection{The January 2007 Simushir tsunami}

The tsunami was generated by a major earthquake $\left(M_{\mathrm{w}}=\right.$ $8.1)$ on 13 January 2007 with epicenter at $46.243^{\circ} \mathrm{N}$, $154.524^{\circ}$ E located on the oceanic slope of the Central Kuril Trench (NGDC Tsunami Database, 2011). This tsunami was weaker that the 2006 Simushir tsunami, but was locally quite intensive (Rabinovich et al., 2008).

The auxiliary source chosen as input for the model was the same as for the 2006 event (an initial circular uplift of $75 \mathrm{~km}$ in diameter and maximum height of $10 \mathrm{~m}$ ). This source center coincided with the earthquake epicenter. The ocean depth at the epicenter was $6877 \mathrm{~m}$. This tsunami was recorded by a smaller number of DART stations than the 2006 tsunami (cf. Laverov et al., 2009); however, one of these stations was DART 21413, which was not in operation during the previous (2006) event. This station was located southward from 


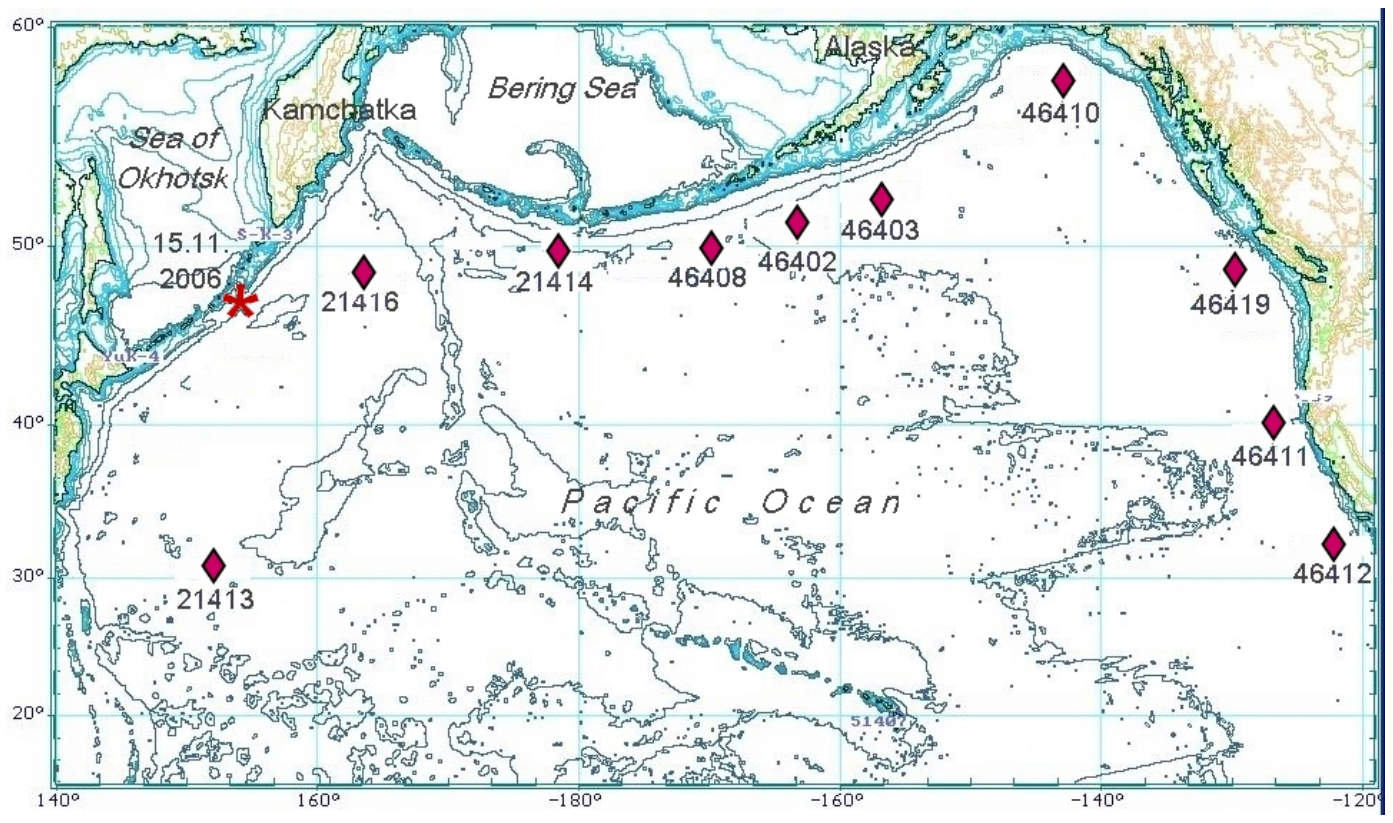

Fig. 4. A map of the North Pacific Ocean showing positions of DART stations and the epicenter of the 2006 Simushir earthquake. Epicenters of the 2007 and 2009 Simushir earthquakes are only slightly shifted from the location of the 2006 epicenter.

the 2007 epicenter (690 miles southeast from Tokyo); during the 2006 event the tsunami was only recorded by DART stations located to the east of the source. It is of additional interest to compare the results for open-ocean stations located in various directions from the source. The corresponding results are shown in Fig. 6.

In general, the agreement between computed and observed waveforms is satisfactory both for DART 46408 near the Aleutian Islands and for DARTs 46419 and 46412 along the US West Coast. Hindcasts based on data from both stations 21414 (eastward from the source) and 21413 (southward from the source) are of almost identical quality. For all cases, the computations show correctly the tsunami manifestation during the initial (negative) phase. The correlation coefficients were within the range $\rho=0.50-0.72$.

\subsection{The January 2009 Simushir tsunami}

The earthquake $\left(M_{\mathrm{w}}=7.4\right)$ occurred on 15 January 2009, approximately in the same region of the Central Kuril Islands as the 2006 and 2007 earthquakes, but it was weaker than the two previous earthquakes. The epicenter of the earthquake was located at $46.857^{\circ} \mathrm{N}, 155.154^{\circ} \mathrm{E}$ (NGDC Tsunami Database, 2011). The earthquake generated a tsunami that was recorded along the coast of the Kuril Islands and by a few DART stations, in particular by DART 21416 located 240 miles from the Kamchatka Peninsula. The latter station was the nearest to the epicenter.

The auxiliary source was taken as a circular uplift of the sea surface $50 \mathrm{~km}$ in diameter and maximum height of $8 \mathrm{~m}$; the source center coincided with the earthquake epicenter.
This source was used as the input for the model. The ocean depth at the epicenter was $6645 \mathrm{~m}$.

The computed and observed tsunami waveforms are shown in Fig. 7. The data from DART 21416 were used to hindcast the waveforms for DART 46408 , located eastward from the source, and for DART 21413, located southward from the source. Similar computations were also made for DARTs 21416 and 46408 based on the data from DART 21413. The computed waveforms were in good agreement with the observed tsunami records for both the area of the Kamchatka Peninsula (point 21416) and the area of the Aleutian Islands (point 46408), especially for the leading waves. The correlation coefficients were within the range $\rho=0.75$ 0.80 .

\subsection{Dependence of the hindcast quality on the diameter of the auxiliary source}

Numerical experiments with auxiliary sources of different diameters $(30,40,50,75$ and $100 \mathrm{~km})$ gave almost identical results. The correlation coefficients between hindcasted and observed waveforms were within the interval of $0.60-0.76$. Smaller values of the correlation coefficients are mostly related to smaller source diameters; this appears to be associated with high-frequency components in simulated wave tails generated by small-scale sources. However, the leading lowfrequency waves were approximated well in all cases. 


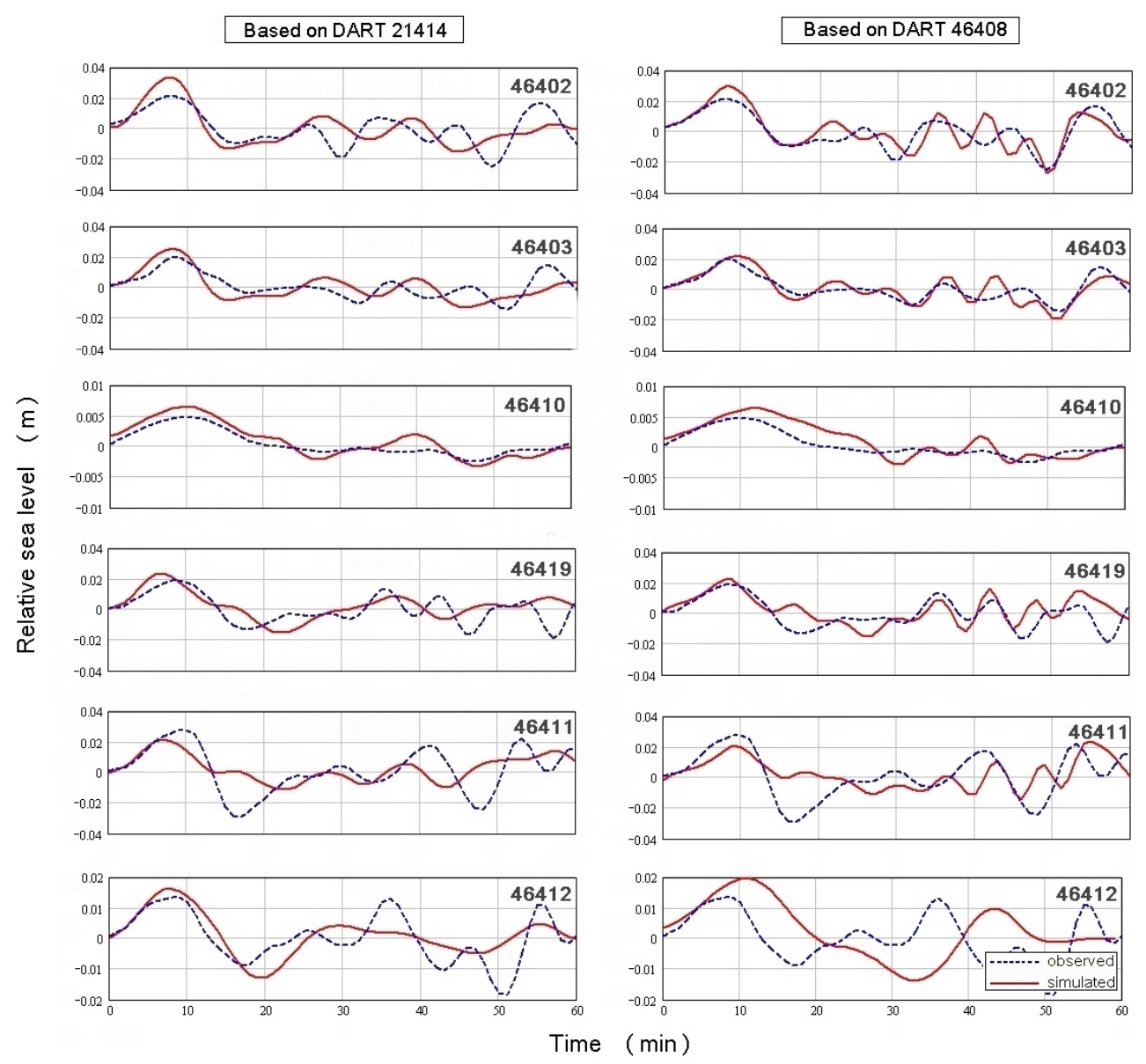

Fig. 5. Computed and observed tsunami waveforms for the 2006 Simushir earthquake for DART sites in the North Pacific Ocean (see Fig. 4 for the DART positions). Left column: using DART 21414 as the reference station; right column: using DART 46408 as the reference station.

\subsection{Results and discussion}

The results of our numerical experiments demonstrate that the diameter of the auxiliary source does not significantly influences the quality of the tsunami forecasting. The best results are achieved (as could be expected) when this diameter is similar to the actual characteristic (transversal) size of the earthquake source area.

In general, the main scope of the present study was to use the observational data from the 2006, 2007 and 2009 Simushir tsunamis to verify the model and this approach. The results are very encouraging. Tsunami forecasts give almost identical results based either on the data from reference DART stations located in the same direction as the target sites (eastward) or from the stations located in other directions (in particular, southward). The results represented in Sects. 4.24.4 confirm the validity of the assumptions made in Sect. 3.1 about this method to evaluate the transfer function describing the evolution of the propagating tsunami waves.
Despite its approximate character, the proposed method can provide the forecast of tsunami wave parameters for any ocean site with sufficiently high quality, and provide realtime operative information for tsunami warning services. It took less than $40 \mathrm{~min}$ to compute the waveforms from the auxiliary source (including the estimation of the transfer function and tsunami forecast for target sites). The tsunami propagation time from the source to the nearest open-ocean station (DART 21414) was about $150 \mathrm{~min}$ for the 2006 and 2007 Simushir events and about $50 \mathrm{~min}$ to DART 21416 for the 2009 event. Thus, taking into account these propagation times and the time needed to identify the tsunami wave in the in situ record (about half of the first wave period), we can estimate the "forecast time" as being approximately $1.5-3 \mathrm{~h}$. Tsunami wave propagation time from the coasts of Japan and Russia to the US West Coast is approximately 9$10 \mathrm{~h}$; that means there are $6-8 \mathrm{~h}$ of advance time before the tsunami reaches that coast. Similarly, for tsunamis generated in the Cascadia Subduction Zone (near the US West Coast), there $6-8 \mathrm{~h}$ of advance time that can be used for the Tsunami 

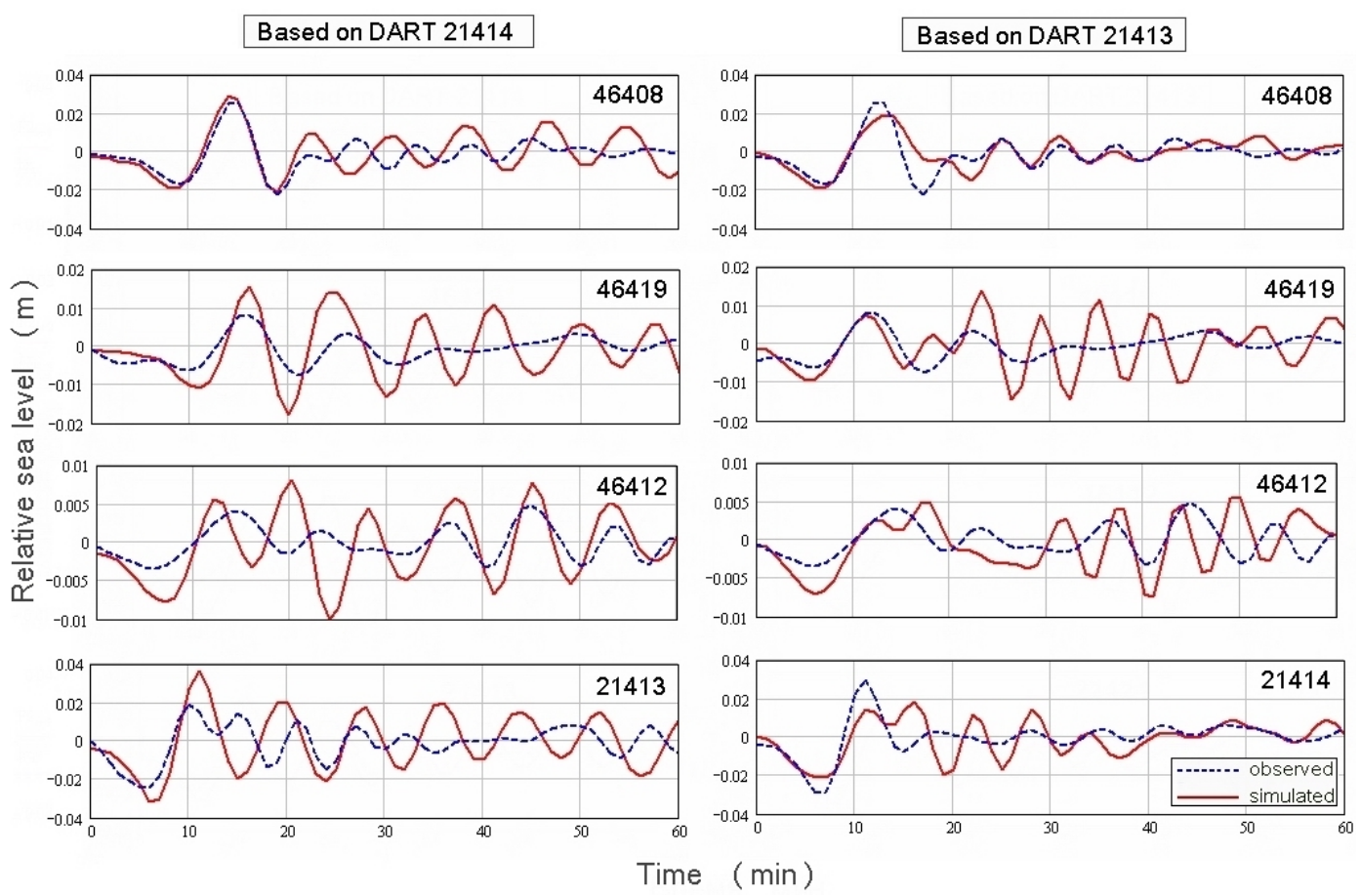

Fig. 6. The same as in Fig. 5 but for the 2007 Simushir tsunami. Left column is for DART 21414 as the reference station; right column is for DART 21413 as the reference station.
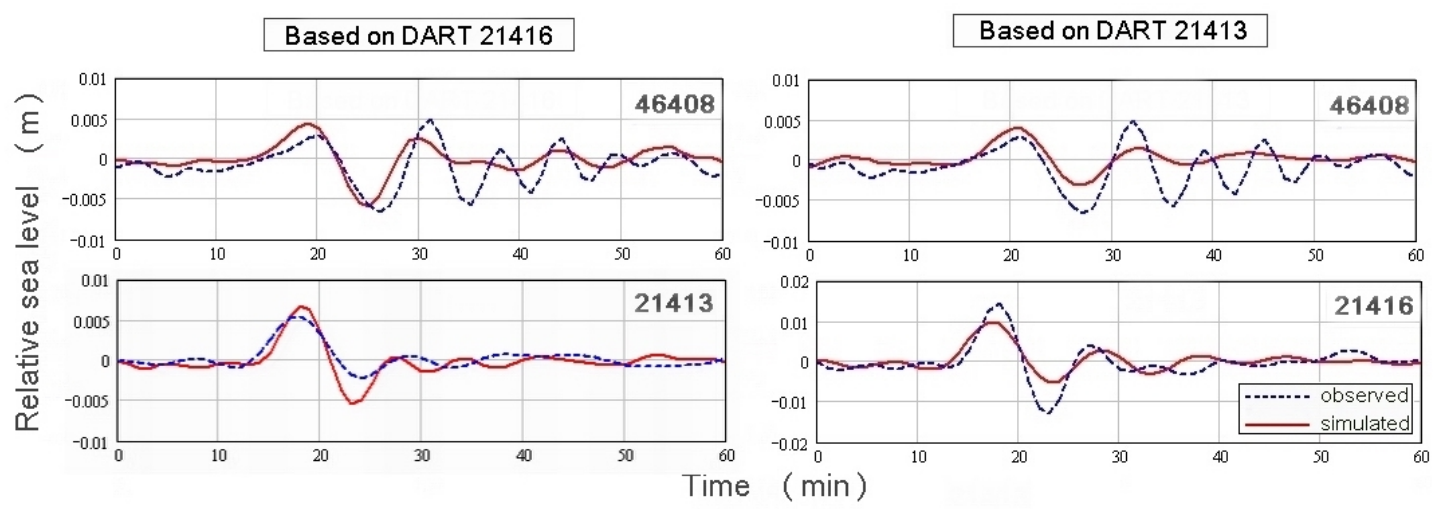

Fig. 7. The same as in Fig. 5 but for the 2009 Simushir tsunami. Left column is for DART 21416 as the reference station; right column is for DART 21413 as the reference station.

Warning on the coasts of Russia and Japan. These simple estimates demonstrate that the proposed method satisfies the requirements of the warning services and can be effectively used for the Tsunami Warning.

The comparison of results presented in this paper with some other results based on assimilation of DART data (cf. Tang et al., 2008; Wei et al., 2008; Titov, 2009) shows that the forecast quality obtained by different methods is comparable. The advantage of the proposed method is that it does not require pre-computed database of synthetic tsunami waveforms. It is especially important for the areas where tsunami early warning systems are just being established and there are no pre-computed databases.
The numerical experiments described in the paper were conducted for open-ocean target sites. For these sites the method is working pretty well. However, the main purpose of TWSs is to provide reliable tsunami forecasts for coastal areas and, if necessary, to declare tsunami alarms for these areas. For this purpose we have to make auxiliary computations (to evaluate transfer functions) using the detailed bathymetry grid (detailed enough to resolve smallscale resonant features of coastal topography). This is a critical problem and we hope that high-resolution coastal topography and bathymetry will allow us to provide the adequate tsunami forecast for the entire coastal zone exposed to arriving tsunami waves. We also expect that this will ensure 
a straightforward forecast not only of the leading tsunami waves but also following tsunami waves which can be much more intensive than the leading waves.

In the present study, the duration of the computed tsunami waveforms did not exceed the duration of the original tsunami records obtained at DART stations. The practical interest is to predict tsunami waveforms of long duration, which would take into account secondary waves reflected from island chains, submarine ridges and mainland coasts. This can be the next step in improvement of the proposed method.

\section{Conclusions}

The reciprocity principle, known in acoustics as an asymptotic assumption, is applied to describe long ocean waves. The similarity conditions for reciprocal sources are obtained. It is shown in numerical experiments that, with a quality sufficient for practical applications, the reciprocity principle Eq. (2), Eq. (3) works quite well even when conditions (a), (b) are not totally satisfied (i.e. when the characteristic time of the wave propagation between the sources is only three to five times greater than the typical wave period, or when the characteristic wavelength exceeds the size of the source area).

The paper presents a method of short-term tsunami forecast using open-ocean sea-level data from distant sites based on the reciprocity principle. It is assumed that the proposed method will enable the computation of a reliable tsunami prediction irrespective of the tsunami generation mechanism. The only additional information required for this method (except an open-ocean in situ record) is the location of the earthquake epicenter. The method enables us to compute expected tsunami waveforms in real-time mode and for any given ocean site.

The method enables the transfer function to be evaluated during the event immediately after obtaining information about the earthquake epicenter location. In contrast to the PMEL/NOAA technology, which is based on a pre-computed Propagation Database that needs updating for newly deployed open-ocean stations, the present method does not require pre-computed tsunami waveforms.

This reciprocity principle method was applied to compute waveforms of the 2006, 2007 and 2009 Simushir tsunamis. The actual tsunami records from open-ocean DART stations were used to verify the method and to estimate the effectiveness of this approach. The computed and observed tsunami waveforms for the regions of the Aleutian Islands and the US West Coast were in good agreement, satisfying the requirements of tsunami warning services. The proposed method can be considered as the basis for creating a program package that can be applied for early tsunami warning for the sites exposed to the tsunami threat. The method can be used for both regional and local tsunami warning services having access to the open-ocean (DART) data in a real-time mode. The method can be applied for newly developing tsunami centers.

Acknowledgements. The author would like to thank Alexei Ivashchenko, Frank González and Issac Fine for their valuable comments and suggestions and Alexander Rabinovich and Fred Stephenson for editing the text.

Edited by: S. Monserrat

Reviewed by: F. Gonzalez and I. Fine

\section{References}

Brekhovskikh, L. M.: Waves in Layered Media, Academic Press, New York, 561 pp., 1960.

Chertock, G.: Transient flexural vibrations on ship-like structures exposed to underwater explosions, J. Acoust. Soc. America, 48, 170-180, 1970.

Chubarov, L. B. and Shokin, Yu. I.: Mathematical modeling in mitigating the hazardous effect of tsunami waves in the ocean, A priori analysis and timely on-line forecast, Sci. Tsunami Hazards, 13, 27-44, 1995.

Chung, J. Y., Kim, S. D., and Ivanov, V. V.: Tsunami wave hindcasting in the Japan Sea, in: Tsunami: Progress in Prediction, Disaster Prevention and Warning, edited by: Tsuchiya, Y. and Shuto, N., Kluwer Academic Publishers, 85-98, 1995.

Fujii, Y. and Satake, K.: Tsunami sources of the November 2006 and January 2007 Great Kuril Earthquakes, Bull. Seism. Soc. Amer., 98, 1559-1571, 2008.

Gusiakov, V. K.: Relationship of tsunami intensity to source earthquake magnitude as retrieved from historical data, Pure Appl. Geophys., 168, 2033-2041, doi:10.1007/s00024-011-0286-2, 2011.

Igarashi, Y., Kong, L., Yamamoto, M., and McCreery, C. S.: Anatomy of historical tsunamis: lessons learned for tsunami warning, Pure Appl. Geophys., 168, 2043-2063, doi:10.1007/s00024-011-0287-1, 2011.

Korolev, Yu. P.: Tsunami numerical modeling for short-term forecasting using data of remote level gauges, Oceanology, 44, 346352, 2004.

Korolev, Yu. P.: The method of the early tsunami warning using data of remote level gauges, in: Proc. 22nd Int. Tsunami Symp., Chania, Crete Island, Greece, 27-29 June 2005, 113-119, 2005.

Korolev, Yu. P.: Retrospective short-term forecast of the 1996 Andreanov (Aleutian Islands) tsunami, Oceanology, 51, 385-393, 2011.

Korolev, Yu. P. and Poplavsky, A. A.: A method of tsunami estimation for short-range forecast service, in: IUGG, XXI General Assembly, Boulder, Colorado, A335, 1995.

Korolev, Yu. and Zaytsev, A.: The numerical model of the early tsunami warning, in: Waves Measurement and Analysis, Proc. Fifth Int. Symp. WAVES 2005, 3-7 July, 2005, Madrid, Spain (on CD), paper \#89, (see also Book of Abstracts, 89-90), 2005.

Landau, L. D. and Lifshits, E. M.: Fluid Mechanics, Vol. 6 (Course of Theoretical Physics), Butterworth-Heinemann, 1987.

Laverov, N. P., Lobkovsky, L. I., Levin, B .W., Rabinovich, A. B., Kulikov, E. A., Fine, I. V., and Thomson, R. E.: The Kuril tsunamis of November 15, 2006, and January 13, 2007 : 
Two trans-Pacific events, Transactions (Doklady) of the Russian Academy of Sciences, Earth Science Section, 426, 386-392, 2009.

Loomis, H. G.: Solution of the linear, long-wave hydrodynamic equations by using unit impulse functions, in: Tsunami Res. Symp. 1974, edited by: Heath, R. A. and Cresswell, M. M., Roy. Soc. New Zealand and UNESCO, Paris, 155-158, 1974.

Loomis, H. G.: Tsunami prediction using the reciprocal property of Green's functions, Mar. Geodesy, 2, 27-39, 1979.

MacInnes, B. T., Pinegina, T. K., Bourgeois, J., Razhigaeva, N. G., Kaistrenko, V. M., and Kravchunovskaya, E. A.: Field survey and geological effects of the 15 November 2006 Kuril tsunami in the middle Kuril Islands, Pure Appl. Geophys., 166, 9-36, 2009.

Miller, G. R.: Relative spectra of tsunamis, Hawaii Inst. Geophys. HIG-72-8, Honolulu, 7 pp., 1972.

Mofjeld, H. O.: Tsunami measurements, in: The Sea, edited by: Bernard, E. N. and Robinson, A. R., Harvard University Press, Cambridge, Vol. 15, 201-235, 2009.

NGDC Tsunami Database: available at: http://www.ngdc.noaa.gov/ hazard/tsu_db.shtml, 2011.

NOAA Magazine: available at: http://www.magazine.noaa.gov/ stories/mag153.htm, 2011.

NOAA NDBC: available at: http://www.ndbc.noaa.gov/dart.shtml, 2011.

Poplavsky, A. A., Khramushin, V. N., Nepop, K. I., and Korolev, Yu. P.: The Operative Tsunami Prediction on the Sea Coasts of the Far East, DVO RAN, Yuzhno-Sakhalinsk, Russia, 1997 (in Russian).

Rabinovich, A. B., Stephenson, F. E., and Thomson, R. E.: The California Tsunami of 15 June 2005 along the coast of North America, Atmos.-Ocean, 44, 415-427, 2006.

Rabinovich, A. B., Lobkovsky, L. I., Fine, I. V., Thomson, R. E., Ivelskaya, T. N., and Kulikov, E. A.: Near-source observations and modeling of the Kuril Islands tsunamis of 15 November 2006 and 13 January 2007, Adv. Geosci., 14, 105-116, doi:10.5194/adgeo-14-105-2008, 2008.

Rayleigh Lord (Strutt, J. W.): The Theory of Sound, Vol. 2, Dover, New York, 1945.
Satake, K.: Inversion of tsunami waveform for the estimation of a fault heterogeneity: Method and numerical experiments, J. Phys. Earth, 35, 241-254, 1987.

Smith, W. H. F. and Sandwell, D. T.: Bathymetric prediction from dense satellite altimetry and sparse shipboard bathymetry, J. Geophys. Res., 99, 803-824, 1994.

Takahasi, R. and Aida, I.: Studies on the spectrum of tsunami, Bull. Earthq. Res. Inst., 39, 523-535, 1961.

Tang, L., Titov, V. V., Wei, Y., Mofjeld, H. O., Spillane, M., Arcas, D., Bernard, E. N., Chamberlin, C., Gica, E., and Newman, J.: Tsunami forecast analysis for the May 2006 Tonga tsunami, J. Geophys. Res., 113, C12015, doi:10.1029/2008JC004922, 2008.

Titov, V. V.: Tsunami forecasting, in: The Sea, edited by: Bernard, E. N. and Robinson, A. R., Harvard University Press, Cambridge, Vol. 15, 367-396, 2009.

Titov, V. V., González, F. I., Bernard, E. N., Eble, M. C., Mofjeld, H. O., Newman, J. C., and Venturato, A. J.: Real-time tsunami forecasting: challenges and solutions, Nat. Hazards, 35, 35-41, 2005.

Uric, R. J.: Principles of Underwater Sound, McGraw-Hill Book Company, Inc, 1975.

Voronina, T. A. and Tcheverda, V. A.: Reconstruction of tsunami initial form via level oscillation, Bull. Novosibirsk Comp. Center, Ser. Math. Meth. Geophys., 4, 127-136, 1998.

Wei, Y., Cheung, K. F., Curtis, G. D., and MsCreery, C. S.: Inverse algorithm for tsunami forecasts, J. Waterw., Ports, Coastal Ocean Eng., ASCE, 129, 60-69, 2003.

Wei, Y., Bernard, E., Tang, L., Weiss, R., Titov, V., Moore, C., Spillane, M., Hopkins, M., and Kânodlu, U.: Realtime experimental forecast of the Peruvian tsunami of August 2007 for U.S. coastlines, Geophys. Res. Lett., 35, L04609, doi:10.1029/2007GL032250, 2008.

Yamazaki, Y., Wei, Y., Cheung, K. F., and Curtis, G. D.: Forecast of tsunamis from the Japan-Kuril-Kamchatka source region, Nat. Hazards, 38, 411-435, 2006. 